\title{
Changes in Knee Joint Space Width in Treatment with a New Hyaluronic-Based Hydrogel
}

\author{
Octav Marius Russu, Andrei Marian Feier*, Tudor Sorin Pop, Marcela Todoran, István Gergely
}

University of Medicine and Pharmacy Tirgu-Mures, Romania

Objective: Our purpose was to assess the effect of a new hyaluronic acid-based (Hymovis $®$ ) injections on joint space width narrowing in patients diagnosed with knee osteoarthritis. Methods: A prospective clinical trial was conducted in the Department of Orthopedics and Traumatology II from the Clinical County Hospital, Tîrgu Mureș, Romania. Thirty-five patients diagnosed with idiopathic knee osteoarthritis received two intraarticular injections with hyaluronic acid-based hydrogel ( $24 \mathrm{mg}$ of hyaluronic acid/3 ml) at one-week interval. Anteroposterior radiographs were obtained before the injections, at six and twelve months after. Minimum joint space width was measured by two senior orthopaedics surgeons at each follow up. Each radiograph was measured again by the same evaluators two weeks apart. Results: Thirty-one patients were present at the final follow-up. A minor reduction in mean weight was noticed (from $82.2 \mathrm{~kg} \pm 16.2 \mathrm{~kg}$ to $80.9 \mathrm{~kg} \pm 16.0, \mathrm{p}>$ 0.398) without any correlation with joint space width narrowing. There were no major changes at the first follow up (6 months) regarding joint space narrowing. A reduction in joint space width was observed however at 12 months varying from $4.4 \mathrm{~mm}(\mathrm{SD} \pm 1.64$, range 1.8-7.1) at the first assessment to $4.3 \mathrm{~mm}$ ( $S D \pm 1.26$, range $0.0-6.8)$ at the final follow-up but with no statistical difference ( $p=0.237)$. Conclusion: No significant modification in joint space width at the final follow-up secondarily proved that two injections of Hymovis $₫$ may slow down narrowing in the knee joint space over a one-year period.

Keywords: joint space width, hyaluronic acid, knee osteoarthritis

Received 18 April 2017 / Accepted 24 May 2017

\section{Introduction}

Knee osteoarthritis (OA) is considered one of the most common degenerative diseases with increasing prevalence in the elderly over the past decades [1]. Studies suggest that $10 \%$ of men and $13 \%$ of women aged 60 or older suffer from symptomatic knee OA [2]. A rise in OA incidence is expected due to an increase of obesity prevalence and a growing elderly population [3]. Even with the increased prevalence there are only a few therapies that may prevent cartilage degeneration. Treatment management comprises a succeeded combination of pharmacological and nonpharmacological alternatives, based on different variables such as age, physical activity, stage of the disease and associated pathology $[4,5]$. One of the common therapies used in the early and mild stages of the disease are intraarticular injections with hyaluronic acid (HA) [5]. Studies revealed that HA leads to a positive and beneficial change within the affected joint by its mechanic effect (lubrication), its chondroprotective mechanism and by acting as a cytokine and anti-inflammatory mediator in the synovial fluid [6-9]. It is also considered that viscosupplementation with hyaluronic acid based products may reduce the time to total knee arthroplasty for up to 8.7 months [10]. Cartilage loss assessment and joint space width (JSW) measurements using serial standardized radiographs are important instruments in the diagnosis, treatment and progression evaluation of knee OA [11-13]. Tibiofemoral JSW measurement is recommended by Osteoarthritis Research So-

* Correspondence to: Andrei Marian Feier

E-mail: andreifeier@gmx.com ciety (OARSI) as the main measurement tool in assessing the biological progression in OA [14]. The United States Food and Drug Administration recommends the use of joint space narrowing (JSN) as a primary evaluation tool in the progression of osteoarthritis in trials with medication that aims to reduce cartilage loss and degeneration. An increase in JSW (i.e. widening of the joints space) at a study endpoint may suggest a new or regrown cartilage and should be the aim of structural outcomes in these trials [15]. The main endpoint used in current clinical trials and large cohort studies is the minimum joint space width $(\mathrm{mJSW})$ and it is considered an indirect measurement tool for cartilage thickness on both medial and lateral compartments of the tibiofemoral joint [14]. A novel biochemical innovation was capable to confer increased viscoelasticity and residence time to natural HA polymers, while maintaining its biotribological characteristics [16]. This innovative technology was implemented in a new molecule "HYADD ${ }^{\circ}$ ", presenting an unprecedented HA-based hydrogel (Hymovis $\left.{ }^{\circ}\right)$ on the market. As it is a new product, its effects on mJSW are not yet extensively described, consequently, our aim was to assess the effect of the new HA derivative Hymovis injections on $\mathrm{mJSW}$ in patients diagnosed with knee OA.

\section{Methods}

A prospective, single-center, clinical trial was conducted in a university hospital (Department of Orthopedics and Traumatology II from the Clinical County Hospital, Tîrgu Mureș, Romania). The study was approved by the local ethical committee and performed in compliance with the 
principles of Good Clinical Practice and the Declaration of Helsinki concerning medical research on humans and the country-specific regulations.

Inclusion criteria were male and female individuals aged 45-80 suffering from idiopathic knee OA for at least 6 months and diagnosed with radiological knee OA Kellgren-Lawrence grade II or III [17]. Exclusion criteria were the following: conditions other than primary idiopathic $\mathrm{OA}$, any type of intra-articular injections in the previous 6 months, heparin or platelet anti-coagulation treatment in the last month, non-steroidal anti-inflammatory drugs (NSAIDs) usage 7 days prior to injection, allergy to HA injections, systemic diseases that may influence the results, presence of any infection or pregnancy and lactation. Out of 79 subjects that were screened and diagnosed with knee $\mathrm{OA}, 41$ matched one or more of our exclusion criteria and were not included in the study. Due to education limitations, three individuals were unable to fill in the forms necessary for enrolment and were excluded. Patients that met our inclusion criteria were informed about our study design and objective and after signing the informed consent they received two consecutive intraarticular injections of $24 \mathrm{mg} / 3 \mathrm{ml}$ of (500-730 kDa) modified HA (Hymovis ${ }^{\circ}$, Fidia Farmaceutici S.p.A, Italy) at one-week interval. As the anterolateral portal proved to be less painful in intraarticular administration of treatments [18], all injections were performed in the outpatient clinic under aseptic conditions in the aforementioned location. Follow-up visits were scheduled at 6 and 12 months after the injections. Conventional digital anteroposterior (AP) radiographs of the tibiofemoral joint were obtained before the infiltration and at each follow-up visit. At 48 hours after each infiltration, every patient was telephoned and questioned about local inflammatory signs, in order to assess the local tolerability and safety. A study nurse was responsible for the patients' evaluation and data collection.

\section{Radiographs JSW analysis}

Standard digital tibiofemoral, weight bearing AP digital radiographs were obtained in full joint extension at the time of enrollment in the study, at six and twelve months after the injections. Standing extended weight bearing radiographs were used in order to achieve maximum standardization and have the ability to properly grade and classify disease advancement. Moreover, in Romania, for economic reasons, the first X-ray examination is made this way in order to assess the type of prosthesis must be prepared in case of future surgery, a type that is influenced by the degree of instability. Also, according to EULAR recommendations, radiographic views are important for optimizing detection of OA features; in particular for the knee, weightbearing and patellofemoral views are recommended [13]. Lequesne's method of measurement was used to determine the joint space narrowing (JSN) [19]. The radiographs were AP aligned and two senior orthopedic surgeons separately and electronically measured the mJSW differences using the OSIRIS image processing software (University Hospital of Geneva). Each physician repeated the radiograph measurements after two weeks. A mean result from the two measurements and assessors was calculated. As the two senior surgeons involved in radiographic evaluations were similar of age and experience and were trained in the same facility under the surveillance of the same supervisor, only inter-rater and intra-rate agreement was assessed.

\section{Statistical Analysis}

GraphPad (InStat) and EpiInfo v 7.1.4.0 (Centers for Disease Control and Prevention, Atlanta, USA) were used for the statistical analysis. mJSW changes from baseline were compared with the outcomes at 12 months post-therapy using Chi-square test and paired $\mathrm{T}$ test. Considering the relatively short follow-up period, a change of $>0.3 \mathrm{~mm}$ on the final radiograph was interpreted as further progression of OA.

\section{Results}

Out of the initial cohort $(\mathrm{n}=35)$ four individuals were missing from the final radiograph evaluation and their results could not be assessed. The final study cohort contained 31 patients. Baseline demographic and patient characteristics are presented in Table I. Usage of NSAIDs involved daily consumption of ibuprofen (up to $1200 \mathrm{mg} /$ day) and/or paracetamol (up to $2 \mathrm{~g} /$ day) or rescue intake of weak opioids (tramadol or codein), paracetamol and/or ibuprofen.

There was no correlation between gender, smoking status, weight and $\mathrm{mJSW}$ changes. However, minor changes were reported regarding patients' characteristics at the final follow-up: a slight decrease in mean weight was observed (from $82.2 \pm 16.2$ to $80.9 \pm 16.0, \mathrm{p}>0.398$ ); and selfreported NSAIDs usage was reduced in 17 patients. OA related characteristics are shown in Table II.

Two patients in the left knee group progressed from OA grade 2 Kellgren and Lawrence (KL) to grade 3 at the end

Table I. Baseline demographic and patient characteristics

\begin{tabular}{lc}
\hline Characteristic & \\
\hline Female, no. (\%) & $21(68)$ \\
Weight, kg, mean \pm SD & $82.2 \pm 16.2$ \\
Age, years, mean \pm SD & $63.2 \pm 8.1$ \\
Smoker $>1$ year, yes, no. (\%) & $12(39)$ \\
Weight bearing workplace, yes, no. (\%) & $16(52)$ \\
Usage of NSAIDs ${ }^{*}$ yes, (\%) & $31(100)$ \\
History of corticosteroid injections $>6$ mo. $^{* *}$, yes, no. (\%) & $6(19)$ \\
\hline
\end{tabular}

"NSAIDs - nonsteroidal anti-inflammatory drugs; ${ }^{\star \star}$ mo. - months

Table II. Disease related characteristics

\begin{tabular}{lcc}
\hline Characteristic & $\begin{array}{c}\text { Left knee } \\
(\mathrm{n}=17)\end{array}$ & $\begin{array}{c}\text { Right knee } \\
(\mathrm{n}=14)\end{array}$ \\
\hline Kellgren and Lawrence grade, no. (\%) & & \\
2 & $8(47)$ & $6(43)$ \\
3 & $9(53)$ & $8(57)$ \\
Morning stiffness, yes, no. (\%) & $13(76)$ & $11(79)$ \\
Medial compartment* & $12(71)$ & $11(79)$ \\
Lateral compartment* $^{*}$ & $5(29)$ & $3(21)$ \\
${ }^{*}$ based on radiographic predominance of cartilage changes &
\end{tabular}

${ }^{*}$ based on radiographic predominance of cartilage changes 
Table III. mJSW at baseline and at each follow-up

\begin{tabular}{|c|c|c|c|c|}
\hline & $\begin{array}{l}\text { mJSW at baseline } \\
\text { (mm, } \pm \text { SD) }\end{array}$ & $\begin{array}{l}\text { mJSW at } 6 \text { months } \\
(\mathrm{mm}, \pm \mathrm{SD})\end{array}$ & $\begin{array}{c}\text { mJSW at } 12 \text { months } \\
(\mathrm{mm}, \pm \mathrm{SD})\end{array}$ & $\begin{array}{l}\text { Average mJSW change } \\
\text { from baseline }{ }^{\star \star \star}\end{array}$ \\
\hline \multicolumn{5}{|c|}{ Mean mJSW* ${ }^{*}$} \\
\hline 2 & $4.7 \pm 1.13$ & $4.7 \pm 1.08$ & $4.5 \pm 1.19$ & $0.23 \mathrm{~mm}$ \\
\hline 3 & $4.2 \pm 2.15$ & $4.2 \pm 2.22$ & $4.1 \pm 1.33$ & $0.18 \mathrm{~mm}$ \\
\hline
\end{tabular}

of the trial. No significant change was noted regarding mJSW at the first follow-up (6 months). A reduction in $\mathrm{mJSW}$ was observed varying from $4.4 \mathrm{~mm}(\mathrm{SD} \pm 1.64$, range 1.8-7.1) at the first assessment to $4.3 \mathrm{~mm}(\mathrm{SD} \pm$ 1.26 , range $0.0-6.8)$ at the final follow-up ( $\mathrm{p}=0.237)$. Inter-rater agreement was $87 \%$ for the first radiographic assessment and $89 \%$ for the second one. Intra-rater error for the two assessments was $5.8 \%$ and $5.6 \%$ respectively. Measurements for each follow up are presented in Table III.

\section{Discussions}

Based on our single-center 12 month follow-up trial results there were no major changes in mJSW in patients treated with the intraarticular hydrogel Hymovis ${ }^{\oplus}$. These stagnant mJSW values might validate the hypothesis that hyaluronic acid based products have a diminishing effect in the progress of knee OA. In a systematic review analyzing different chodroprotective therapies, Gallagher et al. concluded that JSW changes were ameliorated only in 1 out of 3 studies that assessed HA injections compared to placebo [20]. In a case control study, 60 patients were evaluated for radiographic changes (osteophyte, JSW narrowing, joints space area, tibiofemoral angle) and knee pain after intraarticular injections with HA acid [21]. At a mean follow-up of 2.9 years, medial and lateral compartments joint space areas were significantly bigger in case patients compared to controls. However, in the same study, they concluded that mJSW was not influenced by intraarticular infiltrations with HA. In a phase III, 12-month clinical study that evaluated the same product (HYMOVIS ${ }^{\circ}$ ), Benazzo et al. found similar results to ours, with a slight reduction in JSW (from $4.6 \mathrm{~mm}$ at baseline to $4.2 \mathrm{~mm}$ at one-year follow-up. In contrast to our results, they found a significant reduction in morning stiffness $(\mathrm{p}<0.001)$ at 3 months after viscosupplementation [22]. A frequently encountered debate in the literature addresses the molecular weight $(\mathrm{MW})$ of the HA product injected. It was previously demonstrated that HA products with a MW of $500-730 \mathrm{kDa}$ pass easily through the synovial membrane stimulating the endogenous synthesis of HA [23]. It is also specified that this may lead to a large quantity of high molecular $\mathrm{HA}$ to be secreted in the synovial liquid [24]. Also, the quality and quantity of the native HA synthesized by the human synovial fibroblasts is determined by the structure and provenance of the HA existent in the extracellular environment [25]. Rheologi$\mathrm{cal}$ and viscoelastic properties have an essential function in HA products with low molecular weight. Ambrosio et al. managed to obtain a wide range of rheological behaviors on low molecular weight products, from dilute solutions to hydrogels [26]. Our study outcomes reported at one year may be considered limited by some authors, as changes in cartilage and periarticular structures may occur over longer periods in knee OA. However, a stalled advancement of cartilage destruction and disease progression may be recognizable even after shorter periods [27]. Even with the existent synergic results, analgesics and NSAIDs usage was studied as an independent variable in our study due to the fact that our therapy is considered a second-line treatment after selective and non-selective NSAIDs [5]. The lack of a control group is a limitation for the current work and sample size calculation results could not be implemented due to limited finances the relatively small sample of patients. Additionally, due to financial limitations no extra-radiographs could be added to the ones that are standardized for orthopedic assessment in our department. Upcoming trials investigating $\mathrm{mJSW}$ on a screening-based evaluation in larger cohorts with extended follow-up intervals might offer further evidence regarding $\mathrm{OA}$ radiologic progression during this type of treatment.

\section{Conclusions}

There were no major variations in joint space width at the final follow-up, secondarily proving that two injections of Hymovis may slow down narrowing in the knee joint space in a 12-month period. However, a strong encouraging conclusion could not be drawn due to the gradual advancement of radiologic changes characteristic for the disease.

\section{Acknowledgments and funding}

This research was supported by the University of Medicine and Pharmacy of Targu Mures with financial support from S.C. Angelini Pharmaceuticals Romania S.R.L through the grant 15270/03.11.2015.

\section{Conflict of interest}

None to declare.

\section{References}

1. Heidari B. Knee osteoarthritis prevalence, risk factors, pathogenesis and features: Part I. Caspian J Intern Med. 2011 Spring;2(2):205-12.

2. Zhang Y, Jordan JM. Epidemiology of osteoarthritis. Clin Geriatr Med. 2010 Aug;26(3):355-69.

3. Neogi T. The epidemiology and impact of pain in osteoarthritis. Osteoarthritis Cartilage. 2013 Sep;21(9):1145-53.

4. Hochberg MC, Altman RD, April KT, et al - American College of Rheumatology 2012 recommendations for the use of nonpharmacologic and pharmacologic therapies in osteoarthritis of the hand, hip, and knee. Arthritis Care Res. 2012;64: 465-74.

5. McAlindon TE, Bannuru RR, Sullivan MC, et al - OARSI guidelines for the non-surgical management of knee osteoarthritis. Osteoarthritis 
Cartilage 2014;22:363-88.

6. Altman RD, Manjoo A, Fierlinger A, et al - The mechanism of action for hyaluronic acid treatment in the osteoarthritic knee: a systematic review. BMC Musculoskelet Disord. 2015 Oct;16:321.

7. du Souich P. Absorption, distribution and mechanism of action of SYSADOAS. Pharmacol Ther. 2014 Jun;142(3):362-74.

8. Strauss EJ, Hart JA, Miller MD, et al - Hyaluronic acid viscosupplementation and osteoarthritis: current uses and future directions. Am J Sports Med. 2009 Aug;37(8):1636-44.

9. Forsey RW, Fisher J, Thompson J, et al - The effect of hyaluronic acid and phospholipid based lubricants on friction within a human cartilage damage model. Biomaterials. 2006 Sep;27(26):4581-90.

10. Ong $\mathrm{KL}$, Anderson AF, Niazi $F$, et al - Hyaluronic Acid Injections in Medicare Knee Osteoarthritis Patients Are Associated With Longer Time to Knee Arthroplasty. J Arthroplasty. 2016 Aug;31(8):1667-73.

11. Hunter DJ, Zhang YQ, Tu X, et al - Change in joint space width: hyaline articular cartilage loss or alteration in meniscus? Arthritis Rheum. 2006 Aug;54(8):2488-95

12. Crema MD, Nevitt MC, Guermazi A, et al - Progression of cartilage damage and meniscal pathology over 30 months is associated with an increase in radiographic tibiofemoral joint space narrowing in persons with knee OA--the MOST study. Osteoarthritis Cartilage. 2014 Oct;22(10):1743-7.

13. Sakellariou G, Conaghan PG, Zhang W, et al - EULAR recommendations for the use of imaging in the clinical management of peripheral joint osteoarthritis. Ann Rheum Dis. 2017 Apr 7. pii: annrheumdis-2016-210815.

14. Altman R, Brandt K, Hochberg M, et al - Design and conduct of clinical trials in patients with osteoarthritis: recommendations from a task force of the Osteoarthritis Research Society. Results from a workshop. Osteoarthritis Cartilage 1996;4(4):217e43.

15. Draft guidance for industry: clinical development programs for drugs, devices, and biological products for the treatment of osteoarthritis. U.S. Department of Health and Human Services, Food and Drug Administration, Center for Drug Evaluation and Research, Center for Biologics Evaluation and Research, Center for Devices and Radiological Health. 1999. Available from: http://www.fda.gov/cder/guidance/index. htm.

16. Galesso D. An innovative chemistry for intra-articular hyaluronic acid: the MO.RE. ${ }^{\circledR}$ technology, evolution of I.A.H.A therapy. Osteoartrosi.eu 2013;12(3):3-7

17. Kellgren JH, Lawrence JS. Radiological Assessment of Osteo-Arthrosis. Annals of the Rheumatic Diseases. 1957;16(4):494-502.

18. Lee SY. Anterolateral Portal Is Less Painful than Superolateral Portal in Knee Intra-Articular Injection. Knee Surgery \& Related Research. 2015;27(4):228-32.

19. Lequesne M. Quantitative Measurements of Joint Space During Progression of Osteoarthritis: Chrondometry. Keuttner GV, Ed. Rosemont, IL: American Academy of Orthopaedic Surgeons 1995;427e44.

20. Gallagher B, Tjoumakaris FP, Harwood MI, et al - Chondroprotection and the prevention of osteoarthritis progression of the knee: a systematic review of treatment agents. Am J Sports Med. 2015 Mar;43(3):734-44.

21. Oka H, Akune T, Muraki S, et al - The mid-term efficacy of intra-articular hyaluronic acid injections on joint structure: a nested case control study. Mod Rheumatol. 2013 Jul;23(4):722-8.

22. Benazzo F, Perticarini L, Padolino A, et al - A multi-centre, open label, long-term follow-up study to evaluate the benefits of a new viscoelastic hydrogel (Hymovis $\left.{ }^{\circledR}\right)$ in the treatment of knee osteoarthritis. Eur Rev Med Pharmacol Sci. 2016 Mar;20(5):959-68

23. Smith MM. A hexadecylamide derivative of hyaluronan (HYMOVIS®) has superior beneficial effects on human osteoarthritic chondrocytes and synoviocytes than unmodified hyaluronan. J Inflamm (Lond). 2013;10:26.

24. Shimizu C. Histomorphometric and biochemical effect of various hyaluronans on early osteoarthritis. J Rheumatol. 1998 Sep;25(9):1813-

25. Smith MM, Ghosh P. The synthesis of hyaluronic acid by human synovial fibroblasts is influenced by the nature of the hyaluronate in the extracellular environment. Rheumatol Int. 1987;7(3):113-22.

26. Ambrosio L, Borzacchiello A, Netti PA, et al - Rheological study on hyaluronic acid and its derivative solutions. Journal of Macromolecular Science, Part A. 1999;36 (7-8): 991-1000.

27. Kon E1, Mandelbaum B, Buda R, et al - Platelet-rich plasma intraarticular injection versus hyaluronic acid viscosupplementation as treatments for cartilage pathology: from early degeneration to osteoarthritis. Arthroscopy. 2011 Nov;27(11):1490-501. 